\title{
Cumulative/dynamic ROC curve estimation
}

\author{
Pablo Martínez-Camblor \\ Hospital Universitario Central de Asturias (HUCA), Oviedo, Asturies and Universidad \\ Autonoma de Chile, Chile \\ Gustavo F. Bayón \\ Instituto Universitario de Oncología del Principado de Asturias (IUOPA), Asturies \\ Sonia Pérez-Fernández \\ Universidad de Oviedo, Asturies, Spain
}

\section{Summary}

ROC curve is a popular graphical method frequently used in order to study the diagnostic capacity of continuous (bio)markers. When the considered outcome is a time-dependent variable, the direct generalization is known as cumulative/dynamic ROC curve. For a fixed point of time, $t$, one subject is allocated into the positive group if the event happens before $t$ and into the negative group if the event is not happened at $t$. The presence of censored subject, which can not be directly assigned into a group, is the main handicap of this approach. The proposed cumulative/dynamic ROC curve estimator assigns a probability to belong to the negative (positive) group to the subjects censored previously to $t$. The performance of the resulting estimator is studied from Monte Carlo simulations. Some real-world applications are reported. Results suggest that the new estimators provide a good approximation to the real cumulative/dynamic ROC curve.

Key words: Cumulative/dynamic ROC curve; Censored data; Cox regression model; Sensitivity; Specificity.

Email addresses: pablomc@ficyt.es (Pablo Martínez-Camblor), perezsonia@uniovi.es (Sonia Pérez-Fernández) 


\section{Introduction}

The receiver operating-characteristic (ROC) curve [1] is a popular graphical method which, given a diagnostic (bio)marker, displays the false-positive rate (i.e., the inability of the marker to recognize a normal subject, without the studied characteristic, as normal) against the true-positive rate (i.e., the ability of the marker to detect the characteristic of interest, frequently one disease) for all possible thresholds. In addition, the area under the ROC curve (AUC) is frequently used as diagnostic accuracy index. Particularly, in models where the dependent variable is dichotomous, it can be read as a goodness of fit index, for instance, in logistic regression models. In the last decades, both ROC curve and the AUC have received great attention in the specialized literature. There exists a large number of papers which deal with both theoretical and practical aspects of the ROC curve and related problems (see, for instance, Martínez-Camblor [2] for a recent review). The general ROC curve comparison (the manuscripts of Moise, Clement and Raissis [3], Venkatraman and Begg [4], Venkatraman [5], Bandos, Rockette and Gur [6], Braun and Alonzo [7], Martínez-Camblor, Carleos and Corral [8], Krzanowski and Hand [9] and Martínez-Camblor, Carleos and Corral [10] deal with different aspects of this problem from different approaches), ROC curve regression (Cai [11] and Rodríguez-Álvarez et al. [12]), ROC curve for time-dependent events (Heagerty and Zheng [13], Wolf, Schmidt and Ulm [14], among others), meta-analysis of ROC curves (Rutter and Gatsonis [15], Martínez-Camblor [16]) or the study and estimation of its associated cut-off point (Yousef, Kundy and Wagner [17] and Martínez-Camblor [18]) are among the main focuses of interest. Of course, there also exist a number of softwares which perform calculus and figures, just as example, the R package: pROC, ROCR or rocplus, among much others, are freely available in the CRAN (www.r-project.org).

The case where the studied characteristic (disease) is a time-dependent event has also been considered from different approaches. The most direct ROC curve generalization is to reduce the time-dependent event to a dichotomous variable 
for each particular fixed moment $t$. Hence, assuming, without loss of generality, that larger values of the marker, $X$, are associated with higher probabilities of the event, the (cumulative) sensitivity, or true-positive rate, and the (dynamic) specificity, or true-negative rate, are respectively defined by:

$$
\begin{aligned}
& S_{E}^{\mathbb{C}}(x, t)=\mathcal{P}\{X>x \mid T \leq t\}, \\
& S_{P}^{\mathbb{D}}(x, t)=\mathcal{P}\{X \leq x \mid T>t\},
\end{aligned}
$$

where $T$ denotes the time variable. The resulting ROC curve, based on the above cumulative sensitivity and dynamic specificity, is known as cumulative/dynamic $(\mathrm{C} / \mathrm{D})$ ROC curve [4], $\mathcal{R}_{t}^{\mathbb{C} / \mathbb{D}}(\cdot)$. In this approach, all subjects will be used at any fixed time $t$. The $i$-th individual is considered as positive (case) if $t_{i} \leq t$, and as negative (control) if $t_{i}>t$. The cumulative/dynamic ROC curve is appropriate if the predictive model is built by using markers measured at baseline and the researcher is interested in the prognostic properties for a particular (or a small number of) time. With complete information, empirical estimators can be directly defined by,

$$
\begin{aligned}
& \hat{S}_{E}^{\mathbb{C}}(x, t)=\#\left\{x_{i}>x \wedge t_{i} \leq t\right\} / \#\left\{t_{i} \leq t\right\} \\
& \hat{S}_{P}^{\mathbb{D}}(x, t)=\#\left\{x_{i} \leq x \wedge t_{i}>t\right\} / \#\left\{t_{i}>t\right\} .
\end{aligned}
$$

Of course, censored data are the main handicap in order to estimate $\mathcal{R}_{t}^{\mathbb{C} / \mathbb{D}}$. Subjects failing before $t$ or with a follow-up longer than $t$ are directly allocated into the positive and the negative group, respectively. However, it is not clear what to do with those subjects censored before $t$; this is the case of subject D in Figure 1.

Perhaps, in this case, the first temptation is to define an estimator based on the traditional Kaplan-Meier survivor function. However, this estimator, proposed by Heagerty, Lumley and Pepe [19], has serious drawbacks; i) it can take values greater than 1, ii) it can drive to non-monotone sensitivity or specificity functions. Alternatively, in the same paper, the authors also proposed a $\mathcal{R}_{t}^{\mathbb{C} / \mathbb{D}}$ estimator based on the nearest neighbor estimator (KNN) for bivariate 
distributions under random censoring proposed by Akritas [20]; main handicap of this estimator is that the researcher must define a smoothing parameter. Most recently, Wolf, Schmidt and Ulm [14] proposed an estimator based on the Nelson-Aalen cumulative incidence curve (CIC). The use of the Nelson-Aalen estimator avoids the point i) but, as the authors recognize, additional procedures, such as isotonic regression, must be used in order to avoid the point ii).

Other possible time-dependent generalizations for the sensitivity, $S_{E}$, and the specificity, $S_{P}$, have been proposed (see Cai et al. [21] for a recent approach). Particularly, Heagerty and Zheng [13] considered the incident sensitivity defined by Etzioni et al. [22] as

$$
S_{E}^{\mathbb{I}}(x, t)=\mathcal{P}\{X>x \mid T=t\}
$$

to introduce the incident/dynamic (I/D) ROC curve, $\mathcal{R}_{t}^{\mathbb{I} / \mathbb{D}}(p)=S_{E}^{\mathbb{I}}\left(\left[1-S_{P}^{\mathbb{D}}\right]^{-1}(t, p), t\right)$ $(0 \leq p \leq 1)$ where $\left[1-S_{P}^{\mathbb{D}}\right]^{-1}(t, p)=\inf \left\{x:\left[1-S_{P}^{\mathbb{D}}\right](x, t) \leq p\right\}$. From this approach the $i$-th subject is considered as control for $t_{i}>t$ and plays the role of

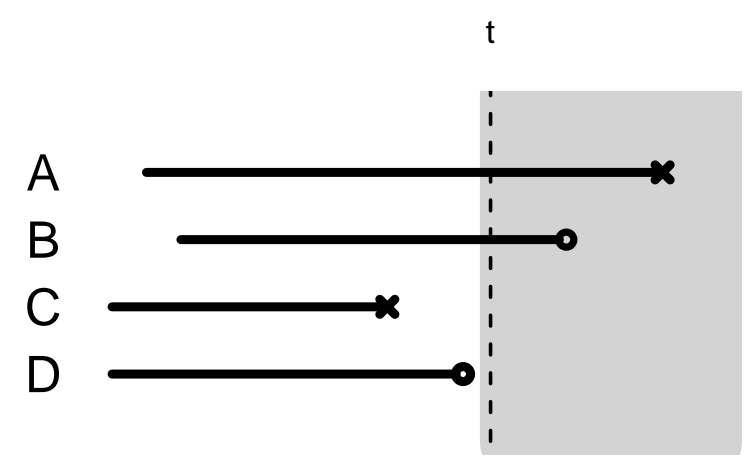

\section{Follow-up}

Figure 1: Schematic situation; A and B are allocated in the negative group, $\mathrm{C}$ in the positive group while D is not directly assigned. 
case when $t_{i}=t$. The I/D ROC curve approach has clear links with the risk functions and, therefore, also with survival hazard models. In addition, the incident sensitivity allows a direct generalization to the case where the considered marker, $X$, is also a time-dependent variable $(=X(t))$. However, it is difficult to interpret without the proportional hazard assumption.

In this paper, the authors are concerned with the $\mathrm{C} / \mathrm{D}$ ROC curve estimation. With this goal, the undefined subjects (those censored before $t$ ) are treated as mixed subjects. They are not completely assigned to a group; but the probability of to be or not be in the group is considered. Rest of the paper is organized as following; in section 2 the proposed estimator is described and its performance is evaluated, via Monte Carlo simulations, in section 3. Section 4 is devoted to real application; particularly, the relationship between the forced ventilatory volume 1 sec. $\left(\mathrm{FEV}_{1}\right)$ and mortality in chronic obstructive pulmonary disease (COPD) patients is studied by using the COllaborative COhorts to assess Multicomponents Indices of COPD in Spain (COCOMICS) dataset (see [23]). In section 5 we present our conclusions. Finally, as appendix, we provide a set of $R$ functions which are useful in order to handle, in practice, the proposed methodology.

\section{Cumulative/dynamic ROC curve estimation}

Conventionally, let $\left\{y_{i}\right\}_{1 \leq i \leq N}=\left\{z_{i}, \delta_{i}, x_{i}\right\}_{1 \leq i \leq N}$ be an independent random sample (with size $N$ ) where for $1 \leq i \leq N, z_{i}$ stands for the observed time; $z_{i}=\min \left\{t_{i}, c_{i}\right\}$, with $t_{i}$ the time to event and $c_{i}$ the censoring time, $\delta_{i}$ is the status $\left(\delta_{i}\right.$ takes the value 1 if $z_{i}=t_{i}$ and 0 if $\left.z_{i}=c_{i}\right)$ and $x_{i}$ stands for the (bio)marker value. From the Bayes theorem, the definition of sensitivity and specificity given in (1) and (2) are equivalent to

$$
\begin{aligned}
& S_{E}^{\mathbb{C}}(x, t)=\frac{\mathcal{P}\{X>x \wedge T \leq t\}}{\mathcal{P}\{T \leq t\}}=\frac{\int \mathcal{P}\{X>x \wedge T \leq t \mid y\} d \boldsymbol{F}_{Y}}{\int \mathcal{P}\{T \leq t \mid y\} d \boldsymbol{F}_{Y}}, \\
& S_{P}^{\mathbb{D}}(x, t)=\frac{\mathcal{P}\{X \leq x \wedge T>t\}}{\mathcal{P}\{T>t\}}=\frac{\int \mathcal{P}\{X \leq x \wedge T>t \mid y\} d \boldsymbol{F}_{Y}}{\int \mathcal{P}\{T>t \mid y\} d \boldsymbol{F}_{Y}},
\end{aligned}
$$


where $y$ is the observed value $(\{z, \delta, x\})$ and $\boldsymbol{F}_{Y}$ its cumulative distribution function (CDF). Note that, in the sampling context, for $i \in\{1, \ldots N\}, \mathcal{P}\{X>$ $\left.x \wedge T \leq t \mid y_{i}\right\}=\mathcal{P}\left\{T \leq t \mid y_{i}\right\} \cdot I_{(x, \infty)}\left(x_{i}\right)\left(I_{A}(x)\right.$ is the indicator function; takes the value 1 if $x \in A$ and 0 otherwise $)$. Then, if $\hat{P}_{i}\left(=\hat{P}_{i}(N)\right)$ is an adequate estimator for $\mathcal{P}\left\{T>t \mid y_{i}\right\}$, the empirical estimators for the sensitivity and the specificity can be written as,

$$
\begin{aligned}
\hat{S}_{E}^{\mathbb{C}}(x, t) & =\frac{\sum_{i=1}^{N}\left(1-\hat{P}_{i}\right) \cdot I_{(x, \infty)}\left(x_{i}\right)}{\sum_{i=1}^{N}\left(1-\hat{P}_{i}\right)}, \\
\hat{S}_{P}^{\mathbb{D}}(x, t) & =\frac{\sum_{i=1}^{N} \hat{P}_{i} \cdot I_{(-\infty, x]}\left(x_{i}\right)}{\sum_{i=1}^{N} \hat{P}_{i}} .
\end{aligned}
$$

Obviously, $z_{i}>t$ implies $\hat{P}_{i}=1$ and an event previous to $t$ implies $\hat{P}_{i}=0$; therefore, for complete information, these estimators are the usual empirical ones. In addition, the consistency of the above estimators is direct if the estimator of $\mathcal{P}\left\{T>t \mid y_{i}\right\}(1 \leq i \leq N)$ has appealing conditions, particularly, that $\left|\hat{P}_{i}-\mathcal{P}\left\{T>t \mid y_{i}\right\}\right| \rightarrow_{N} 0$ in probability. On the other hand, it is obvious that the resulting $\mathrm{C} / \mathrm{D}$ ROC curve overcomes the drawbacks reported by the Kaplan-Meier based C/D ROC curve: for a fixed point $t$, it is monotone and always takes values below or equal to 1 .

Different procedures can be used in order to estimate the above probability in those subjects which are not absolutely defined. We proposed a semiparametric one; by using a proportional hazard Cox regression model; and a non-parametric proposal; by using directly the Kaplan-Meier estimator. From the proportional hazard Cox regression model we can estimate the hazard function $\lambda(t)=\lambda_{0}(t) \exp \{\beta \cdot X\}$, this quantity allows to compute $\hat{P}_{i}=\hat{S}(t \mid X=$ $\left.x_{i}\right) / \hat{S}\left(z_{i} \mid X=x_{i}\right)$, where $\hat{S}$ is the survival function estimated from the Cox regression model. Unfortunately, due to $X$ is usually a continuous variable, for the Kaplan-Meier method we can not directly estimate the probability for one particular value $X=x_{i}$ and this condition is replaced by $X \leq x_{i}$; then we select values satisfying $X \leq x_{i}$ and compute the Kaplan-Meier estimator to obtain $\hat{P}_{i}=\hat{S}_{K M}(t) / \hat{S}_{K M}\left(z_{i}\right)$, where $\hat{S}_{K M}$ is the survival function estimated by the Kaplan-Meier method referred to those subjects satisfying $X \leq x_{i}$. 
Indeterminate or mixed subjecs play a fundamental role. Note that an absolute uncertainty about the situation of the $i$ th subject at time $t\left(\hat{P}_{i}=1 / 2\right)$ implies that, when this subject is allocated into a group, we will commit an error with a probability of $1 / 2$, with independence of the group in which it will be allocated; in these cases, the capacity diagnostic of the studied biomarker will be limited.

In order to illustrate the problem, we have considered a real problem. Particularly, we have considered a dataset which contains information about 863 (= $N)$ kidney transplant patients. This data, free available within the $\mathrm{R}$ package KMsurv, has been previously used with the same goal by Wolf, Schmidt and Ulm [14]. Information about time to death and age of patients (in this case used as a mortality marker) were collected. Interested reader is referred to Klein and Moeschberger [24] for more details about the data. Figure 2 depicts the situation for the first five subjects when the considered time is 9 years: the patient with $\mathrm{ID}=3$ died the seventh year, hence it is within the positive group; patients 4 and 5 were still alive at ninth year, therefore, they are within the negative group. However, the real situation for patients 1 and 2 are unknown. The probability that those patients are still alive nine years after the follow-up can be estimated by using the Kaplan-Meier estimator. Particularly, for the first subject, we must make the estimation considering only the subjects with age smaller than 46 resulting the estimation $\hat{P}_{1}=0.88 / 0.95(=0.86)$. Similarly, for the second subject, considering only the subjects with age smaller than 51 , it is obtained the value $\hat{P}_{2}=0.91$; in the proposed model, both are considered as mixed subjects.

Figure 3 depicts different ROC curve estimations. At left, in order to make the usual ROC curve $\left(D_{I}\right)$; the unclassified subjects, called mixed, were removed and a total of 140 positives and 17 negatives were finally considered. Both the Kaplan-Meier $\left(K_{M}\right)$ and the $\operatorname{KNN}\left(A_{K}\right)$ based estimations for $\mathcal{R}_{t}^{\mathbb{C} / \mathbb{D}}(t=9$ yrs. $)$ are also depicted (computed by using the $\mathrm{R}$ package: survivalROC). Observed differences between the $A_{K}$ and the CIC estimations were negligible when the 
span parameter (related with the KNN method) is close to zero. At right, the same usual ROC curve (plotted as references); and the C/D ROC curve estimations by using the method proposed above based on both the proportional hazard Cox regression $\left(N_{C}\right)$ and the non-parametric Kaplan-Meier estimator $\left(N_{K}\right)$.

\section{Simulation study}

In order to study the practical behaviour of the proposed methodology, a Monte Carlo simulation study was carried out. Similarly to Heatherty and Zheng [13], the joint distribution of $\{\log ($ Time $)$, Marker $\}$ is a standard bivariate normal distribution with correlation coefficient $\rho$ (cases $-1 / 4$ and $-3 / 4$ were studied). In addition, two different sample sizes $(N=100,200)$ were explored. The considered distribution for the censoring time, $\log (C)$, was also normal with standard deviation 1 and mean $0(\mathcal{P}\{C<T\}=1 / 2)$ and 1.19 $(\mathcal{P}\{C<T\}=1 / 5)$. The censoring time was drawn independently of the time

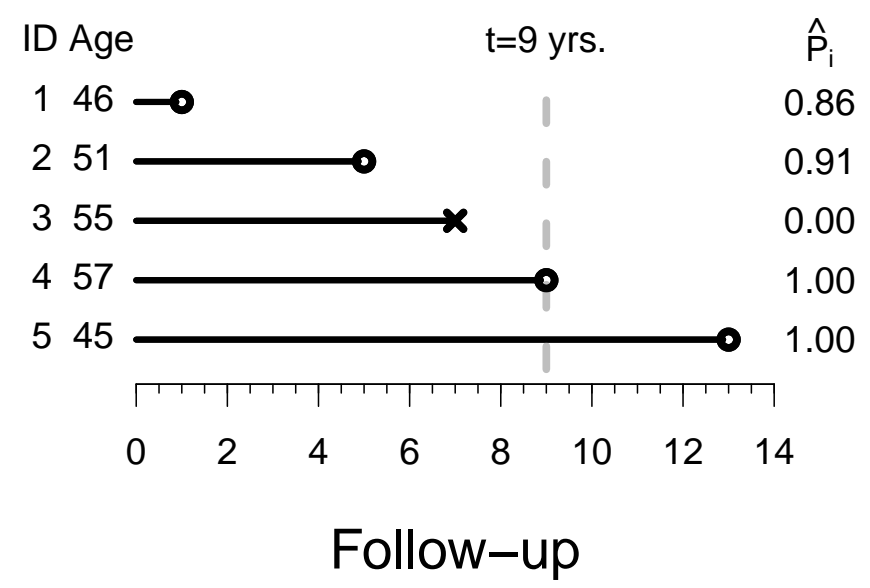

Figure 2: Schematic situation for IDs 1 to 5 of the kidney transplantation data. 
to event, but not of (bio)marker values, which are related in such a way that $\mathbb{E}[\log (C) \cdot M]=\tau($ cases $\tau=0,1 / 4$ were considered $) . \mathrm{C} / \mathrm{D}$ ROC curve estimations for $\log ($ Time $)=-1,0,1$ were computed based on the Kaplan-Meier estimator $\left(K_{M}\right)$, the KNN method with span $=0.01 \cdot N^{-1 / 5}\left(A_{K}\right)$, the direct method, which removes the subjects with incomplete information, called mixed subjects in this paper $\left(D_{I}\right)$, and by using the proposed estimator based on both Cox regression $\left(N_{C}\right)$ and Kaplan-Meier estimator $\left(N_{K}\right)$. Figure 4 depicts the theoretical ROC curves from where the samples were drawn.

Table 1 shows the mean \pm standard deviation for $0.01 \cdot \sqrt{N} \cdot \int_{0}^{1} \mid \hat{\mathcal{R}}(p)-$ $\mathcal{R}(p) \mid d p$, where $\mathcal{R}$ is the real C/D ROC curve and $\hat{\mathcal{R}}$ is its estimation when $\tau=0$ (censoring time and (bio)marker values are drawn independently). Five studied methods performed similarly. Proposed $N_{C}$ statistics obtained better results than $N_{K}$ (note that in the simulated scenario it is satisfied the proportional hazard assumption) and it was the best of all in most cases. As expected, the observed differences between the proposed method and the other ones were
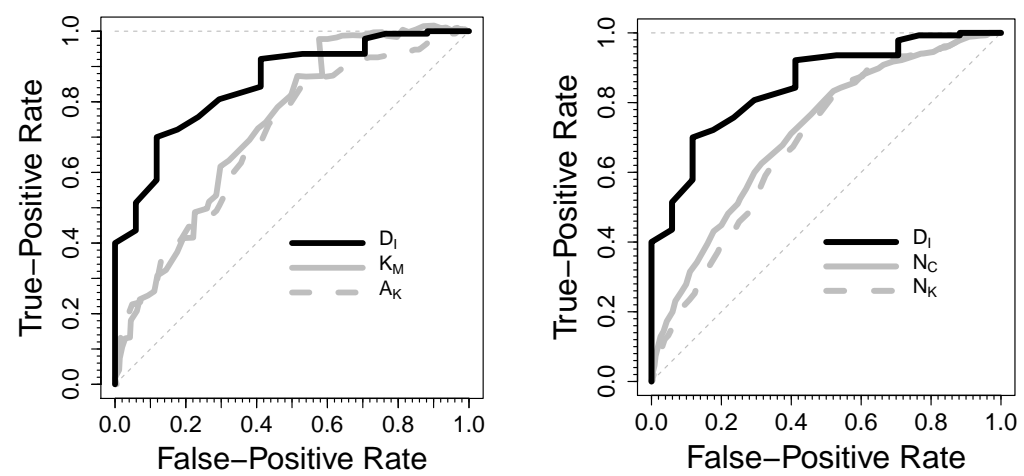

Figure 3: At left, direct ROC curve estimation ( $D_{I}$; removing the mixed subjects), KaplanMeier $\left(K_{M}\right)$ and $\mathrm{KNN}\left(A_{K}\right.$; with span=0.01 $\left.N^{-1 / 5}\right)$ based cumulative/dynamic ROC curve estimations. At right, $D_{I}$ (as references) and proposed estimations based on both Cox regression $\left(N_{C}\right)$ and Kaplan-Meier $\left(N_{K}\right)$. 
clearer when the censored percentages were larger, particularly, for $\% \mathrm{C}=50$ and $\log (t)=1$, the proposed methodology achieved the best results.

Table 2 is the same as table 1 but considering that $\tau=1 / 4$. Observed results were similar to the previous obtained ones. It seems that five considered estimators are robust respect to this correlation configuration between the (bio)marker and censoring time.

\section{Real-data application: the COCOMICS study}

The proposed methodology is applied to study the capacity of forced ventilatory volume 1 sec. $\left(\mathrm{FEV}_{1}\right)$ to predict mortality in chronic obstructive pulmonary disease (COPD) patients. With this goal, we consider the data of the COllaborative COhorts to assess Multicomponent Indices of COPD in Spain (COCOMICS study). This dataset included 11 Spanish cohorts with a total of 3,633 patients out of a total of $15,878.17$ people per year. The interested reader is referred to Soriano et al. [25] and to Marin et al. [23] for complete information about the data. Figure 5 (up-left) depicts the Kaplan-Meier estimation with a $95 \%$ confidence interval for the COCOMICS data and the number of patients at risk at $1,4,7,10$ and 13 years.
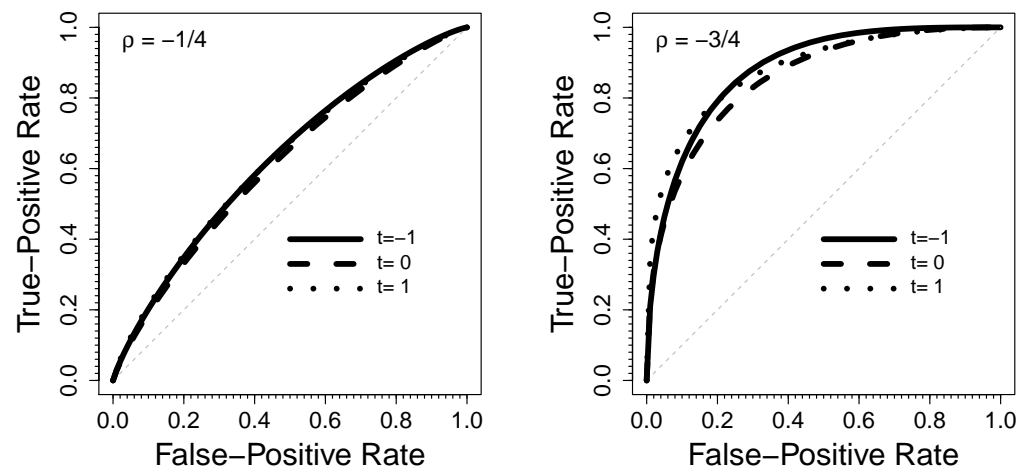

Figure 4: Real ROC curves from where the samples were drawn. 
Table 1: Mean \pm standard deviation of $0.01 \cdot \sqrt{N} \cdot \int_{0}^{1}|\hat{\mathcal{R}}(p)-\mathcal{R}(p)| d p$, where $\mathcal{R}$ is the real $\mathrm{C} / \mathrm{D}$ ROC curve and $\hat{\mathcal{R}}$ is its estimation, computed from 5,000 Monte Carlo iterations for $\tau=0$.

\begin{tabular}{|c|c|c|c|c|c|c|c|c|}
\hline$N$ & $\rho$ & $\% \mathrm{C}$ & $\log (t)$ & $K_{M}$ & $A_{K}$ & $D_{I}$ & $N_{C}$ & $N_{K}$ \\
\hline \multirow[t]{3}{*}{100} & $-1 / 4$ & $20 \%$ & -1 & $0.834 \pm 0.376$ & $0.833 \pm 0.399$ & $0.827 \pm 0.365$ & $0.828 \pm 0.373$ & $0.830 \pm 0.375$ \\
\hline & & & 0 & $0.613 \pm 0.271$ & $0.614 \pm 0.289$ & $0.625 \pm 0.294$ & $0.587 \pm 0.266$ & $0.591 \pm 0.267$ \\
\hline & & & 1 & $0.934 \pm 0.463$ & $0.920 \pm 0.460$ & $0.887 \pm 0.443$ & $0.767 \pm 0.392$ & $0.767 \pm 0.415$ \\
\hline \multirow[t]{3}{*}{100} & $-3 / 4$ & $20 \%$ & -1 & $0.476 \pm 0.214$ & $0.474 \pm 0.248$ & $0.471 \pm 0.214$ & $0.468 \pm 0.214$ & $0.471 \pm 0.214$ \\
\hline & & & 0 & $0.424 \pm 0.182$ & $0.429 \pm 0.212$ & $0.410 \pm 0.179$ & $0.390 \pm 0.173$ & $0.399 \pm 0.181$ \\
\hline & & & 1 & $0.591 \pm 0.320$ & $0.623 \pm 0.362$ & $0.486 \pm 0.283$ & $0.411 \pm 0.182$ & $0.476 \pm 0.176$ \\
\hline \multirow[t]{3}{*}{100} & $-1 / 4$ & $50 \%$ & -1 & $0.868 \pm 0.391$ & $0.866 \pm 0.420$ & $0.853 \pm 0.374$ & $0.801 \pm 0.368$ & $0.835 \pm 0.385$ \\
\hline & & & 0 & $0.744 \pm 0.338$ & $0.745 \pm 0.358$ & $0.750 \pm 0.350$ & $0.585 \pm 0.299$ & $0.614 \pm 0.310$ \\
\hline & & & 1 & $1.519 \pm 0.866$ & $1.092 \pm 0.542$ & $1.123 \pm 0.453$ & $0.813 \pm 0.483$ & $0.956 \pm 0.646$ \\
\hline \multirow[t]{3}{*}{100} & $-3 / 4$ & $50 \%$ & -1 & $0.532 \pm 0.231$ & $0.492 \pm 0.250$ & $0.489 \pm 0.216$ & $0.448 \pm 0.211$ & $0.481 \pm 0.221$ \\
\hline & & & 0 & $0.570 \pm 0.243$ & $0.533 \pm 0.264$ & $0.487 \pm 0.215$ & $0.365 \pm 0.171$ & $0.466 \pm 0.243$ \\
\hline & & & 1 & $1.511 \pm 1.065$ & $1.051 \pm 0.667$ & $1.118 \pm 1.009$ & $0.391 \pm 0.181$ & $0.761 \pm 0.490$ \\
\hline \multirow[t]{3}{*}{200} & $-1 / 4$ & $20 \%$ & -1 & $0.821 \pm 0.356$ & $0.807 \pm 0.381$ & $0.831 \pm 0.387$ & $0.816 \pm 0.354$ & $0.818 \pm 0.356$ \\
\hline & & & 0 & $0.618 \pm 0.267$ & $0.612 \pm 0.286$ & $0.630 \pm 0.297$ & $0.591 \pm 0.260$ & $0.595 \pm 0.261$ \\
\hline & & & 1 & $0.950 \pm 0.442$ & $0.952 \pm 0.472$ & $0.997 \pm 0.496$ & $0.784 \pm 0.371$ & $0.784 \pm 0.398$ \\
\hline \multirow[t]{3}{*}{200} & $-3 / 4$ & $20 \%$ & -1 & $0.484 \pm 0.201$ & $0.499 \pm 0.247$ & $0.481 \pm 0.201$ & $0.478 \pm 0.201$ & $0.481 \pm 0.201$ \\
\hline & & & 0 & $0.435 \pm 0.181$ & $0.435 \pm 0.208$ & $0.424 \pm 0.177$ & $0.400 \pm 0.171$ & $0.412 \pm 0.179$ \\
\hline & & & 1 & $0.596 \pm 0.258$ & $0.618 \pm 0.328$ & $0.539 \pm 0.245$ & $0.428 \pm 0.181$ & $0.532 \pm 0.285$ \\
\hline \multirow[t]{3}{*}{200} & $-1 / 4$ & $50 \%$ & -1 & $0.866 \pm 0.380$ & $0.856 \pm 0.407$ & $0.879 \pm 0.412$ & $0.801 \pm 0.358$ & $0.835 \pm 0.374$ \\
\hline & & & 0 & $0.749 \pm 0.334$ & $0.750 \pm 0.359$ & $0.784 \pm 0.379$ & $0.589 \pm 0.300$ & $0.621 \pm 0.313$ \\
\hline & & & 1 & $1.599 \pm 0.882$ & $1.284 \pm 0.638$ & $1.453 \pm 0.703$ & $0.859 \pm 0.509$ & $0.995 \pm 0.671$ \\
\hline \multirow[t]{3}{*}{200} & $-3 / 4$ & $50 \%$ & -1 & $0.535 \pm 0.224$ & $0.525 \pm 0.264$ & $0.498 \pm 0.209$ & $0.456 \pm 0.213$ & $0.494 \pm 0.219$ \\
\hline & & & 0 & $0.581 \pm 0.238$ & $0.529 \pm 0.247$ & $0.536 \pm 0.223$ & $0.375 \pm 0.170$ & $0.412 \pm 0.263$ \\
\hline & & & 1 & $1.278 \pm 0.755$ & $1.177 \pm 0.689$ & $1.173 \pm 0.642$ & $0.457 \pm 0.220$ & $1.042 \pm 0.517$ \\
\hline
\end{tabular}


Table 2: Mean \pm standard deviation of $0.01 \cdot \sqrt{N} \cdot \int_{0}^{1}|\hat{\mathcal{R}}(p)-\mathcal{R}(p)| d t$, where $\mathcal{R}$ is the real C/D ROC curve and $\hat{\mathcal{R}}$ is its estimation, computed from 5,000 Monte Carlo iterations for $\tau=1 / 4$.

\begin{tabular}{|c|c|c|c|c|c|c|c|c|}
\hline$N$ & $\rho$ & $\% \mathrm{C}$ & $\log (t)$ & $\boldsymbol{K}_{M}$ & $A_{K}$ & $D_{I}$ & $N_{C}$ & $N_{K}$ \\
\hline \multirow[t]{3}{*}{100} & $-1 / 4$ & $20 \%$ & -1 & $0.828 \pm 0.375$ & $0.829 \pm 0.402$ & $0.829 \pm 0.370$ & $0.825 \pm 0.375$ & $0.830 \pm 0.376$ \\
\hline & & & 0 & $0.601 \pm 0.270$ & $0.614 \pm 0.294$ & $0.614 \pm 0.301$ & $0.588 \pm 0.269$ & $0.604 \pm 0.277$ \\
\hline & & & 1 & $0.867 \pm 0.421$ & $0.913 \pm 0.446$ & $0.914 \pm 0.407$ & $0.754 \pm 0.384$ & $0.823 \pm 0.424$ \\
\hline \multirow[t]{3}{*}{100} & $-3 / 4$ & $20 \%$ & -1 & $0.468 \pm 0.207$ & $0.465 \pm 0.238$ & $0.470 \pm 0.207$ & $0.466 \pm 0.205$ & $0.468 \pm 0.205$ \\
\hline & & & 0 & $0.411 \pm 0.179$ & $0.421 \pm 0.196$ & $0.415 \pm 0.182$ & $0.389 \pm 0.167$ & $0.399 \pm 0.169$ \\
\hline & & & 1 & $0.532 \pm 0.291$ & $0.536 \pm 0.281$ & $0.522 \pm 0.334$ & $0.398 \pm 0.169$ & $0.412 \pm 0.208$ \\
\hline \multirow[t]{3}{*}{100} & $-1 / 4$ & $50 \%$ & -1 & $0.830 \pm 0.382$ & $0.872 \pm 0.420$ & $0.845 \pm 0.364$ & $0.803 \pm 0.372$ & $0.855 \pm 0.396$ \\
\hline & & & 0 & $0.657 \pm 0.394$ & $0.748 \pm 0.354$ & $0.741 \pm 0.339$ & $0.582 \pm 0.299$ & $0.672 \pm 0.345$ \\
\hline & & & 1 & $1.253 \pm 0.698$ & $1.054 \pm 0.495$ & $1.078 \pm 0.409$ & $0.779 \pm 0.462$ & $1.123 \pm 0.657$ \\
\hline \multirow[t]{3}{*}{100} & $-3 / 4$ & $50 \%$ & -1 & $0.509 \pm 0.235$ & $0.484 \pm 0.239$ & $0.494 \pm 0.225$ & $0.450 \pm 0.206$ & $0.482 \pm 0.205$ \\
\hline & & & 0 & $0.563 \pm 0.257$ & $0.499 \pm 0.223$ & $0.493 \pm 0.235$ & $0.368 \pm 0.168$ & $0.421 \pm 0.189$ \\
\hline & & & 1 & $1.271 \pm 0.909$ & $0.698 \pm 0.388$ & $1.240 \pm 1.041$ & $0.343 \pm 0.162$ & $0.539 \pm 0.369$ \\
\hline \multirow[t]{3}{*}{200} & $-1 / 4$ & $20 \%$ & -1 & $0.822 \pm 0.359$ & $0.818 \pm 0.384$ & $0.832 \pm 0.381$ & $0.820 \pm 0.359$ & $0.824 \pm 0.361$ \\
\hline & & & 0 & $0.605 \pm 0.266$ & $0.614 \pm 0.289$ & $0.638 \pm 0.305$ & $0.595 \pm 0.266$ & $0.612 \pm 0.275$ \\
\hline & & & 1 & $0.870 \pm 0.402$ & $0.938 \pm 0.452$ & $1.029 \pm 0.510$ & $0.758 \pm 0.374$ & $0.836 \pm 0.422$ \\
\hline \multirow[t]{3}{*}{200} & $-3 / 4$ & $20 \%$ & -1 & $0.474 \pm 0.203$ & $0.493 \pm 0.246$ & $0.476 \pm 0.204$ & $0.472 \pm 0.202$ & $0.474 \pm 0.201$ \\
\hline & & & 0 & $0.430 \pm 0.188$ & $0.430 \pm 0.202$ & $0.433 \pm 0.189$ & $0.406 \pm 0.173$ & $0.418 \pm 0.176$ \\
\hline & & & 1 & $0.561 \pm 0.264$ & $0.547 \pm 0.258$ & $0.559 \pm 0.288$ & $0.423 \pm 0.174$ & $0.433 \pm 0.202$ \\
\hline \multirow[t]{3}{*}{200} & $-1 / 4$ & $50 \%$ & -1 & $0.831 \pm 0.371$ & $0.859 \pm 0.405$ & $0.881 \pm 0.410$ & $0.803 \pm 0.364$ & $0.855 \pm 0.392$ \\
\hline & & & 0 & $0.674 \pm 0.307$ & $0.753 \pm 0.364$ & $0.822 \pm 0.406$ & $0.593 \pm 0.305$ & $0.692 \pm 0.356$ \\
\hline & & & 1 & $1.294 \pm 0.691$ & $1.222 \pm 0.570$ & $1.360 \pm 0.619$ & $0.818 \pm 0.493$ & $1.217 \pm 0.721$ \\
\hline \multirow[t]{3}{*}{200} & $-3 / 4$ & $50 \%$ & -1 & $0.535 \pm 0.230$ & $0.497 \pm 0.228$ & $0.499 \pm 0.206$ & $0.451 \pm 0.188$ & $0.488 \pm 0.192$ \\
\hline & & & 0 & $0.633 \pm 0.274$ & $0.515 \pm 0.213$ & $0.518 \pm 0.221$ & $0.385 \pm 0.172$ & $0.444 \pm 0.193$ \\
\hline & & & 1 & $1.129 \pm 0.678$ & $0.786 \pm 0.414$ & $0.931 \pm 0.797$ & $0.391 \pm 0.186$ & $0.625 \pm 0.442$ \\
\hline
\end{tabular}


In this dataset, differences between the five considered $\mathrm{C} / \mathrm{D}$ ROC curve estimations at 10 years were really small (see Figure 5, top-right). The proposed estimator based on Kaplan-Meier showed itself more conservative than the other ones. At this point, 1,212 subjects were classified as positives and 198 were allocated into the negative group.

The prediction capacity of the (bio)marker (measured from the area under the ROC curve, AUC) was high along the follow-up time. It was about 0.73 at six months to decrease until 0.68 at two years (lowest value); after that, the diagnostic capacity increases until 0.76 at fourteen years. The integrated AUC along the follow-up time was 0.72. Figure 5 (down) depicts the AUC evolution with a $95 \%$ confidence interval (based on bootstrap replications).
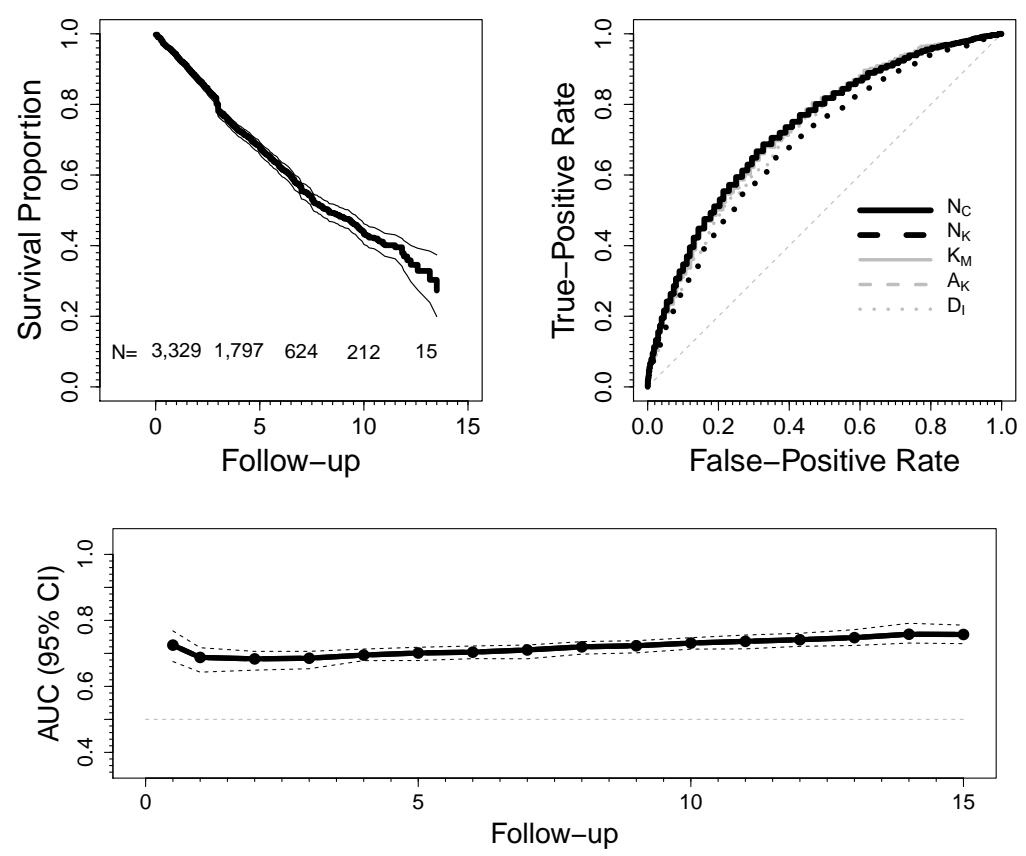

Figure 5: Kaplan-Meier survival curve estimation, top-left; C/D ROC curve estimation at ten years by using the five considered methods, top-right; AUC evolution with a $95 \%$ confidence interval, down. 


\section{Conclusions}

In this paper the authors deal with the cumulative/dynamic ROC curve estimation in the presence of right-censored data. The proposed methodology assigns a probability of belonging to the negative group (respectively to the positive group) to those patients whose real status at considered point remains unknown (those censored before the considered point). This probability can be estimated from different methods; in this paper a semi-parametric (based on Cox regression) and a non-parametric (based on Kaplan-Meier) ones are considered. However, other methods can be used with this goal, even methods adapted to particular situations, ranging from competing-risk or multi-state contexts, to the non-monotone relationship ROC curve generalization [26]. The proposed estimator avoids the drawbacks of the previously existing ones; $i$ ) it is monotone and always ranges between 0 and 1, ii) it does not depend on smooth parameters. In the real-data example, the five considered estimators performed similarly, but simulation suggests that both $N_{C}$ and $N_{K}$ perform well and they are always better than the previous ones when the observed percentage of censorship is high. When some correlation between the (bio)marker and the censoring time is added, obtained results do not change substantially.

It should be noted that the direct method, removing the undefined subjects, usually obtains good results. However, do not use these individuals is not a good methodological practice; we are removing a particular subset of individuals which can have some interesting properties. For instance, if there exists some kind of relationship between the considered marker and the censoring time, the results will be strongly biased.

Perhaps, the main flaws in the presented methodology is the lack of a rigorous study of its theoretical properties. However, the proposed estimator has two different parts; the first one is the traditional ROC curve for complete data, and the second one is to assign, to each mixed subject, the probability that the event has not happened before the fixed point $t$. This probability depends 
on several factors which are not previously clear. At this point, it is good to remark again the good results provided by the simulation study.

Finally, as appendix, we provide some $\mathrm{R}$ functions which compute the proposed methodology. The authors expect that a suitable $\mathrm{R}$ package will be soon available on the CRAN.

\section{Acknowledgements}

The authors are grateful to the COCOMICS group for the permission to use the data.

\section{Appendix: $R$ functions}

The following $\mathrm{R}$ functions make the computes and display plots for the proposed C/D ROC curve estimator. The used notation is direct.

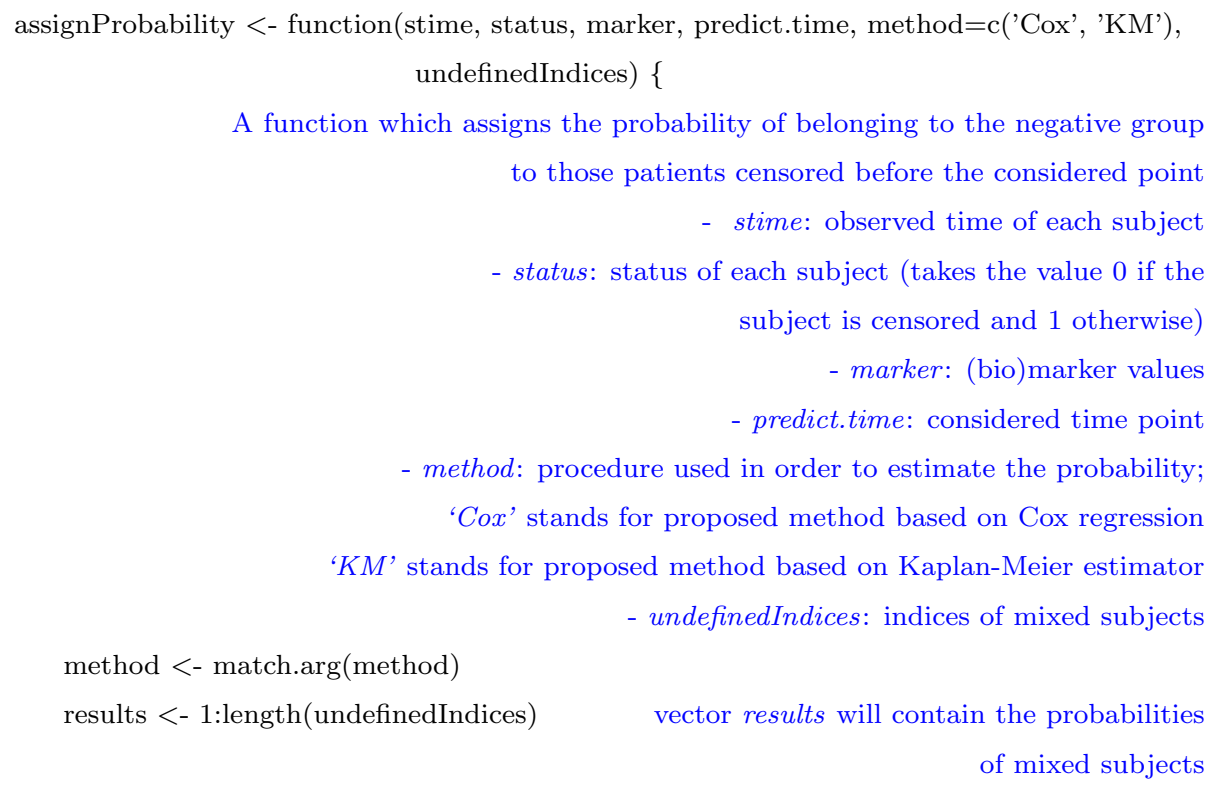

Proposed method based on Cox regression

if $(\operatorname{method}==$ 'Cox') \{

fit $<-\operatorname{coxph}(\operatorname{Surv}($ stime, status $) \sim$ marker $)$ 
fits a Cox proportional hazard regression model whose covariate is marker, i.e., $h(t)=h_{0}(t) \cdot e^{\beta X}$ md <- survfit(fit, newdata=data.frame(cbind(stime,status,marker $))$ ) survival curves from the previously fitted Cox model for $(\mathrm{j}$ in 1:length(undefinedIndices)) for each mixed subject \{ f <- approxfun(c(min(md $\$$ time $)-1, \operatorname{md} \$$ time, $\max (\operatorname{md} \$$ time $)+1)$, $\mathrm{c}(1, \operatorname{md} \$ \operatorname{surv}[$, undefinedIndices[j]], 0))

$f$ constains a list of points which linearly interpolate given data points, so it is an approximation of the survival function estimated from the

Cox regression model results $[\mathrm{j}]<-\mathrm{f}($ predict.time $) / \mathrm{f}($ stime$[$ undefinedIndices $[\mathrm{j}]])$

probability considered in mixed subjects based on

Cox regression model if (is.na(results[j] ) \{ results $[\mathrm{j}]<-1$

if results $[j]$ is a missing value, it takes the value 1 , so the $j$-th mixed subject is assigned to the negative group \}

\}

Proposed method based on Kaplan-Meier estimator

\} else if (method $==$ 'KM') \{

for ( $\mathrm{j}$ in 1 :length(undefinedIndices)) for each mixed subject \{ $\operatorname{idx}<-$ which $($ marker $<=$ marker[undefinedIndices[j]])

$i d x$ constains the indices of those subjects whose marker is smaller than or equal to the marker of the considered mixed subject fit $<-\operatorname{survfit}(\operatorname{Surv}(\operatorname{stime}[\mathrm{idx}]$, status$[\mathrm{idx}]) \sim 1)$ survival curve from Kaplan-Meier estimator using only the previous subjects $\mathrm{f}<-$ stepfun(fit\$time, c(1, fit\$surv))

interpolating step function from survival curve above results $[\mathrm{j}]<-\mathrm{f}($ predict.time $) / \mathrm{f}($ stime[undefinedIndices $[\mathrm{j}]])$

probability considered in mixed subjects based on Kaplan-Meier estimator

if (is.na(results[j] )) \{

results $[\mathrm{j}]<-1$

if results [j] is a missing value, it takes the value 1 , so the $j$-th mixed subject is assigned to the negative group 


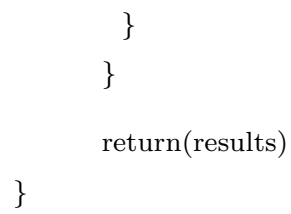


both sensitivity and specificity vectors will have

specificity $<-$ cutPoints

for (i in 1:length(cutPoints))

\{

sensitivity[i] <- $(\operatorname{sum}($ marker[positiveIndices] $>$ cutPoints[i] $)+$

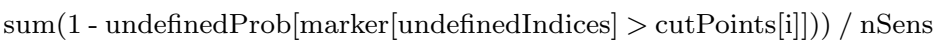

sensitivity estimation from expression in page 6

specificity $[\mathrm{i}]<-(\operatorname{sum}(\operatorname{marker}[$ negativeIndices $]<=\operatorname{cutPoints}[\mathrm{i}])+$ sum(undefinedProb[marker[undefinedIndices] $<=$ cutPoints[i]])) $/$ nSpec

specificity estimation from expression in page 6

\}

rocFunction <- approxfun(1 - specificity, sensitivity)

rocFunction contains a list of points which linearly interpolate given data

points of the cumulative/dynamic ROC curve estimation

auc $<$ - integrate(rocFunction, 0,1 )

AUC estimation by adaptive quadrature of rocFunction over the unit interval results $<-\operatorname{list}(\mathrm{TP}=$ sensitivity, $\mathrm{TN}=$ specificity, undefinedProb=undefinedProb,

cutPoints $=$ cutPoints, auc $=$ auc $\$$ value, aucAbsError $=$ auc $\$$ abs.error,

predict.time $=$ predict.time, method $=$ method $)$

output:

- TP: sensitivity estimation, true positive rate

- $T N$ : specificity estimation, true negative rate

- undefinedProb: probability of belonging to the negative group to those mixed subjects

- cutPoints: thresholds considered

- auc: AUC, the final estimate of the integral above

- aucAbsError: estimation of the modulus of the absolute error from integral estimation above

- predict.time: considered time point

- method: procedure used in order to estimate the probability considered; it can takes the value "Cox" or "KM"

attr(results, 'class') $<-$ 'cdroc'

return(results)

return outputs above 
cdroc $<$ - function(stime, status, marker, predict.time, method $=\mathrm{c}($ 'Cox', 'KM'), ci=FALSE,

$$
\text { boot.n=100, conf.level }=0.95 \text {, seed }=2032)\{
$$

A function which returns outputs of singleCdroc function above, including some aspects about a conf.level confidence interval (if $c i$ is TRUE) for the area under the cumulative/dynamic ROC curve

- boot.n: number of bootstrap replications considered - seed: seed considered (for reproducibility)

method <- match.arg(method)

if (ci) $\{\quad$ if the user is interested in calculating confidence interval set.seed(seed) specify the seed sampledIndices <- sapply(1:boot.n, function(xx) sample(length(stime), length(stime), replace $=$ TRUE) sampledIndices is a matrix containing the indices of each bootstrap replication allResults <- lapply(1:boot.n, function(index, sampledIndices) \{

currentIndices <- sampledIndices[, index] singleCdroc(stime[currentIndices], status[currentIndices], marker[currentIndices], predict.time, method) \}, sampledIndices $=$ sampledIndices) apply the function singleCdroc to each bootstrap sample result <- singleCdroc(stime, status, marker, predict.time, method) apply the function singleCdroc to the main sample allAucs <- sapply(allResults, function(xx) xx\$auc) vector allAucs contains AUC estimations of each bootstrap sample result $\$$ meanAuc $<-$ mean(allAucs) mean of AUC estimations result \$ciAuc <- quantile(allAucs, c(1 - conf.level, conf.level)) conf.level confidence interval for AUC

result $\$$ ci $<-$ TRUE

result\$boot.n $<-$ boot.n

result $\$$ conf.level $<-$ conf.level

result \$seed $<-$ seed

result\$aucs $<-$ allAucs

return(result) return outputs above \} else \{ if the user is not interested in calculating confidence interval return(singleCdroc(stime, status, marker, predict.time, method))

only return outputs of singleCdroc function \} \} 


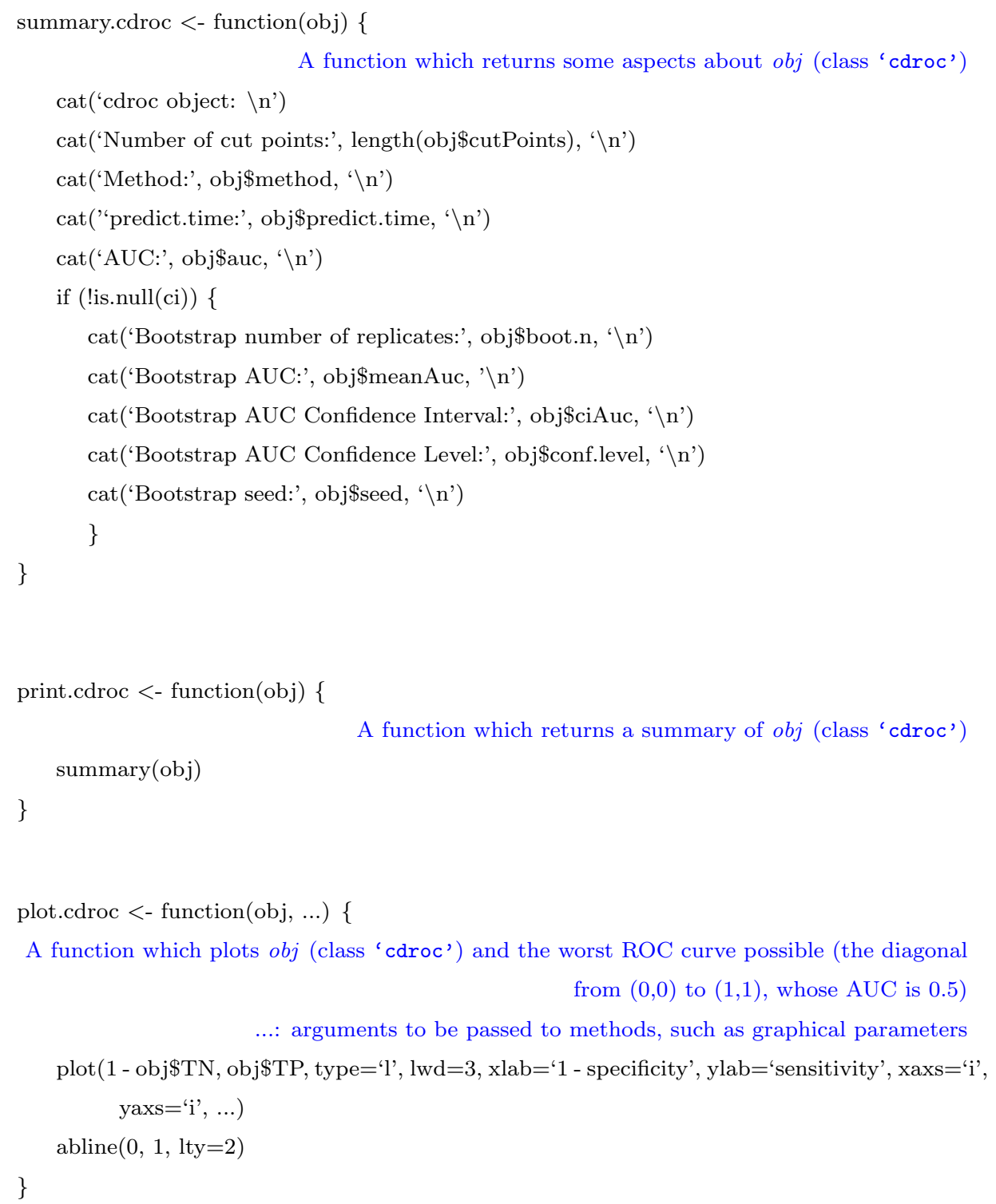

\section{References}

[1] Green DM, Swets JA, Signal detection theory and psychophysics, 1966, New York: Wiley.

[2] Martínez-Camblor P, Area under the ROC curve comparison in the presence of missing data, Journal of the Korean Statistical Society, 2013, 42(4), 431-442. 
[3] Moise A, Clement B, Raissis M, A test for crossing receiver operating characteristic (ROC) curves, Communications in Statistics-Theory and Methods, 1988, 17, 1985-2003.

[4] Venkatraman ES, Begg CB, A distribution-free procedure for comparing receiver operating characteristic curves from a paired experiment, Biometrika, 1996, 83(4), 835-848.

[5] Venkatraman ES, A permutation test to compare receiver operating characteristic curves, Biometrics, 2000, 56, 1134-1138.

[6] Bandos AI, Rockette HE, Gur D, A permutation test sensitive to differences in areas for comparing ROC curves from paired design, Statistics in Medicine, 2005, 24, 2873-2893.

[7] Braun T, Alonzo TA, A modified sign test for comparing paired ROC curves, Biostatistics, 2008, 9, 364-372.

[8] Martínez-Camblor P, Carleos C, Corral N, Powerful nonparametric statistics to compare $k$-independent ROC curves, Journal of Applied Statistics, 2011, 38(7), 1317-1332.

[9] Krzanowski WJ, Hand DJ, Testing the difference between two KolmogorovSmirnov values in the context of receiver operating characteristic curves, Journal of Applied Statistics, 2011, 38(3), 437-450.

[10] Martínez-Camblor P, Carleos C, Corral N, General nonparametric ROC curves comparison, Journal of the Korean Statistical Society, 2013, 42, 71-81.

[11] Cai T, Semi-parametric ROC regression analysis with placement values, Biostatistics, 2004, 5(1), 45-60.

[12] Rodríguez-Álvarez MX, Tahoces PG, Cadarso-Suárez C, Lado MJ, Comparative study of ROC regression techniques-Applications for the computed-aided diagnostic system in breast cancer detection, Computational Statistics and Data Analysis, 2011, 55(1), 888-902.

[13] Heagerty P, Zheng Y, Survival model predictive accuracy and ROC curves, Biometrics, 2005, 61(1), 92-105. 
[14] Wolf P, Schmidt G, Ulm K, The use of ROC for defining the validity of the prognostic index in censored data, Statistics and Probability Letters, 2011, 81, 783-791.

[15] Rutter C, Gatsonis C, A hierarchical regression approach to meta-analysis of diagnostic test accuracy evaluations, Statistics in Medicine, 2001, 20, 2865-2884.

[16] Martínez-Camblor P, Fully non-parametric ROC curve estimation for randomeffects meta-analysis, 2014, Statistical Methods in Medical Research.

[17] Yousef WA, Kundu S, Wagner RF, Nonparametric estimation of the threshold at an operating point on the ROC curve, Computational Statistics and Data Analysis, 2009, 33(12), pp. 4370-4383.

[18] Martínez-Camblor P, Nonparametric cutoff point estimation for diagnostic decisions with weighted errors, Revista Colombiana de Estadística, 2011, 34(1), 133-146.

[19] Heagerty PJ, Lumley T, Pepe MS, Time-dependent ROC curves for censored survival data and a diagnostic marker, Biometrics, 2000, 56, 337-344.

[20] Akritas MG, Nearest neighbor estimation of a bivariate distribution under random censoring, Annals of Statistics, 1994, 22(3), 1299-1327.

[21] Cai T, Pepe MS, Zheng Y, Lumley T, Jenny NS, The sensitivity and specificity of markers for event times, Biostatistics, 2006, 7(2), 182-197.

[22] Etzioni R, Pepe MS, Longton G, Hu C, Goodman G, Incorporating the time dimension in receiver operating characteristic curves: A case study of prostate cancer, Medical Decision Making, 1999, 19, 242-251.

[23] Marin JM, Alfageme I, Almagro P, Casanova C, Esteban C, Soler-Cataluña JJ, de Torres JP, Martínez-Camblor P, Miravitlles M, Celli BR, Soriano J, Multicomponent indices to predict mortality in COPD: the COCOMICS study, European Respiratory Journal, 2013, 42, 323-332.

[24] Klein JP, Moeschberger ML, Survival analysis: Techniques for censored and truncated data, 2003, New York: Springer. 
[25] Soriano J, Alfageme I, Almagro P, Casanova C, Esteban C, Soler-Cataluña JJ, de Torres JP, Martínez-Camblor P, Miravitlles M, Celli BR, Marin JR, Distribution and prognostic validity of the new global initiative for chronic obstructive lung disease grading classification, Chest, 2013, 143(3), 694-702.

[26] Martínez-Camblor P, Corral N, Rey C, Pascual J, Cernuda-Morollón E, ROC curve generalization for non-monotone relationships, Statistical Methods in Medical Research, 2014, doi:10.1177/0962280214541095. 